\title{
Engineering Carbon Nanotubes and Nanotube Circuits Using Electrical Breakdown
}

\author{
Philip G. Collins, Michael S. Arnold, Phaedon Avouris*
}

\begin{abstract}
Carbon nanotubes display either metallic or semiconducting properties. Both large, multiwalled nanotubes (MWNTs), with many concentric carbon shells, and bundles or "ropes" of aligned single-walled nanotubes (SWNTs), are complex composite conductors that incorporate many weakly coupled nanotubes that each have a different electronic structure. Here we demonstrate a simple and reliable method for selectively removing single carbon shells from MWNTs and SWNT ropes to tailor the properties of these composite nanotubes. We can remove shells of MWNTs stepwise and individually characterize the different shells. By choosing among the shells, we can convert a MWNT into either a metallic or a semiconducting conductor, as well as directly address the issue of multiple-shell transport. With SWNT ropes, similar selectivity allows us to generate entire arrays of nanoscale field-effect transistors based solely on the fraction of semiconducting SWNTs.
\end{abstract}

Carbon nanotubes exhibit several technologically important characteristics. Metallic (m) nanotubes can carry extremely large current densities $(1,2)$; semiconducting (s) nanotubes can be electrically switched on and off as field-effect transistors (FETs) $(3,4)$. The two types may be joined covalently $(5,6)$. Unfortunately, no methods exist for reliably preparing only $\mathrm{m}$ or $\mathrm{s}$ nanotubes (or nanotube junctions) either by selective synthesis or through post-synthesis separation. This lack of control, compounded by nanotubes' tendency to bundle together, has been seen as the primary block to nanotube-based electronic technology.

Here we demonstrate a simple and reliable method for permanently modifying MWNTs and SWNT ropes to tailor the properties of these bundled nanotubes (7). Our approach uses current-induced electrical breakdown to eliminate individual shells one at a time. Carbon nanotubes can withstand remarkable current densities, exceeding $10^{9} \mathrm{~A} / \mathrm{cm}^{2}$, in part due to their strong carbon-carbon bonding. However, at high enough currents nanotubes ultimately fail. In MWNTs, this failure occurs in air at a certain threshold power through the rapid oxidation of the outermost carbon shell (8). Because the oxidation of defect-free graphite can proceed only at extremely high temperatures, it was concluded that the primary factor in the breakdown initiation was current-induced defect formation and that self-heating plays only a secondary role.

We have used this process to break cur-

IBM T. J. Watson Research Center, Yorktown Heights, NY 10598, USA.

*To whom correspondence should be addressed. Email: avouris@us.ibm.com rent-carrying nanotubes selectively without affecting parallel nanotubes carrying little or no current. For example, the current distribution in a MWNT favors the outermost shell because it is in direct contact with the external electrodes. This distribution can leave the innermost shells carrying little or no current, which protects them during current-induced oxidation. In a bundle or SWNT rope, the distribution of current is more uniform because many individual nanotubes can simultaneously be in good contact with the external electrodes. Generally, there is no reason for some SWNTs to be favored over others, but we can use an electrostatically coupled gate electrode to select the s-SWNTs and deplete them of their carriers. Once depleted, the s-SWNTs are protected from damage in the same way as the inner shells of a MWNT, and high current densities can be used to initiate oxidation of the metallic SWNTs.

The removal of carbon shells from these composite conductors can be observed both electrically and with the use of microscopy. Electrically, the breakdown of a single carbon shell results in a partial conductance drop that typically occurs within a few milliseconds. When stressed at sufficiently high bias, multiple independent drops occur as one carbon shell after another is broken. The resulting staircase of current as a function of time is shown in Fig. 1A, as eight shells of a MWNT are sequentially removed. Either atomic force or scanning electron microscopy can resolve the gradual thinning of both MWNTs and SWNT ropes (Fig. 1, B and C). Using the former method, MWNT diameters can be determined before and after thinning; the change in apparent diameter has a linear correspondence with the number of breakdown steps observed.
On a practical level, this controlled destruction allows us to effectively separate semiconducting SWNTs from mixtures of SWNTs and to easily generate nanotubebased FETs. More fundamentally, the process can allow insight into the complex electronic structure and transport properties of MWNTs and SWNT ropes. By removing the stress at the occurrence of individual conductance steps, these composite nanowires may be recharacterized upon the loss of each constituent conductor. Multiple complementary transport measurements can, for example, probe deeper and deeper into the inner shells of a MWNT so that transport through its different shells can be compared. Here, we will first describe representative experimental results obtained with a MWNT, and then address SWNT devices.

The different shells of a MWNT are presumed to alternate in a random fashion between $\mathrm{m}$ and s states (9). We directly tested this assumption by using controlled breakdown followed by low-bias or low-temperature measurements, which are believed to probe only the outermost shell of a MWNT $(10,11)$. As in previous measurements on SWNTs $(3,4)$, we differentiate between sand $\mathrm{m}$-shells by measuring the conductance $G$ as a function of gate voltage $V_{\mathrm{g}}$ using a relatively small, $10-\mathrm{mV}$ source-drain bias. An $\mathrm{m}$ shell is characterized by a $G$ that is independent of $V_{\mathrm{g}}$ or nearly so, whereas an s shell can be electrostatically depleted of carriers by the gate.

Figure 2 shows the room temperature $G\left(V_{\mathrm{g}}\right)$ for various different layers within a particular MWNT. Initially, this 9.5-nm diameter m-MWNT had $\mathrm{n}$ shells. Figure 2A shows the strong modulation in $G\left(V_{\mathrm{g}}\right)$ observed after removing three of the outermost shells. Removing a fourth layer resulted in an $\mathrm{m} G\left(V_{\mathrm{g}}\right)$, and removing yet another produced another s $G\left(V_{\mathrm{g}}\right)$. We interpret this variation as a signature of the alternating character of the carbon shells being removed.

$G$ did not fall to zero for a particular s-shell solely because of the contribution of inner $\mathrm{m}$-shells that remain conducting. Note that the depletion minimum of the $G\left(V_{\mathrm{g}}\right)$ curve for shell $n-3$ coincides with the conductance of the underlying shell n-4. In this case, the outer, s-shell (n-3) could be fully depleted by the gate, but the measured conductance included leakage through the underlying mshell. Additional measurements show that this leakage is energetically frozen out in the low-temperature, low-bias limit [in agreement with previous suggestions (12)], indicating that the intershell coupling is thermally activated.

With further breakdown of this MWNT, we can reach the limit of very few, very small diameter shells. Upon removing the tenth carbon shell, the MWNT began to behave 


\section{REPOR T S}

like a perfect, intrinsic FET with a region of zero conductance even at room temperature due to the complete depletion of carriers (Fig. 2B). Similar characteristics were found for individual s-SWNTs $(3,4)$, although the SWNTs used were strongly p-type (i.e., hole carriers) and did not have symmetric $G\left(V_{\mathrm{g}}\right)$ characteristics. The complete depletion of the MWNT indicates that no m-shells remain, and this behavior persisted until the 14th carbon shell was removed, at which time the MWNT circuit finally opened. On the basis of the known intershell spacing of nearly 0.34 nm $(13,14)$, a MWNT of this diameter can have at most 14 shells, in agreement with our shell-by-shell count.

A striking feature of Fig. $2 \mathrm{~B}$ is the gradual increase of the zero conductance region as the final s-shells are removed. The width of this region is proportional to the band gap of the semiconductors, with conduction above and below the gap due to electron and hole carriers, respectively (15). A remarkable characteristic of s-carbon nanotubes is that their band gap energy is inversely proportional to their diameter
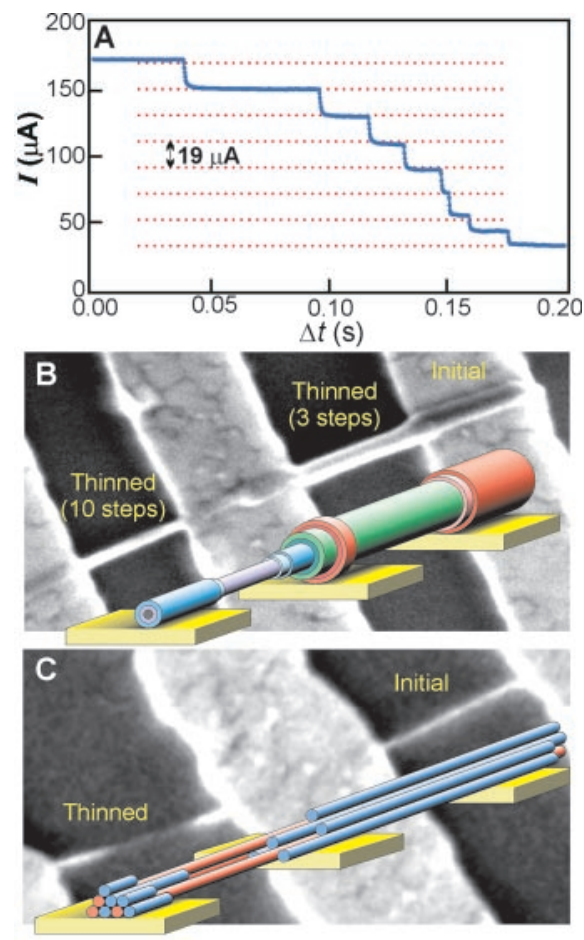

Fig. 1. (A) The partial electrical breakdown of a MWNT at constant voltage stress proceeds in a series of discrete steps corresponding to the loss of individual carbon shells from the MWNT. Equally spaced dotted lines emphasize the surprisingly regular spacing. (B) Images of partially broken MWNTs show clear thinning, with a decrease in radius equal to the intershell spacing $(0.34 \mathrm{~nm})$ times the number of completed breakdown steps. The two segments of this sample were independently thinned by 3 and 10 shells, as depicted by the color overlays. (C) Similar thinning, as observed in SWNT ropes.
$(16,17)$, so that progressively smaller carbon shells should in fact exhibit larger band gaps. If we consider only the initial MWNT diameter and the intershell spacing, we can estimate the diameter of each carbon shell and calculate the expected ratios between band gaps of the innermost shells. As shown in Table 1, the calculated ratios agree well with the experimental ratios, as defined by the conductance peaks on either side of the conductance gap.

Next, we discuss the electrical characteristics of the same 9.5-nm MWNT in the opposite, high-bias regime. Figure $3 \mathrm{~A}$ shows a sequence of high-bias current-voltage characteristics $(I-V \mathrm{~s})$ effectively characterizing the MWNT with $n, n-1, n-2$, etc. shells until only a single shell remains (18). Each $I-V$ curve shows unmistakable current saturation at a steadily increasing bias similar to that observed in individual SWNTs (19) but at much higher currents. The removal of each shell from the MWNT appeared to decrease this saturation level by a fixed amount of $\sim 20 \mu \mathrm{A}$, in agreement with Fig. 1A. This step-by-step decrease indicates that at high bias all of the MWNT shells contributed to transport. Furthermore, the series of $I-V$ curves shows the bias necessary to bring each subset of shells into current saturation.

Besides the decrease of the current saturation value, the sequence of $I-V$ curves in Fig. 3 also shows increasing nonlinearity as shells are removed. A semilog plot of selected $I-V \mathrm{~S}$ shows the trend from a linear $I-V$

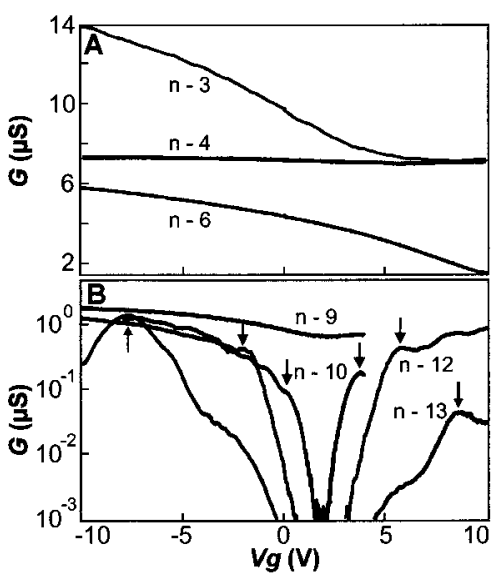

Fig. 2. By halting the stress on each breakdown event, a MWNT may be characterized after the loss of each constituent shell. (A) The low-bias conductance $G\left(V_{g}\right)$ changes between $\mathrm{s}$ and $\mathrm{m}$ behaviors due to the changing nature of the outermost shell at each stage of breakdown. (B) When the last metallic shell (labeled n-9) has been removed, the remaining semiconducting shells can be completely depleted to give regions of zero conductance. Taking the indicated peaks (arrows) in $G\left(V_{g}\right)$ to correspond to the conduction and valence band edges, we can determine the electronic band gaps of different shells within a constant of proportionality. toward an exponential characteristic of the form $I=\mathrm{A} \exp \left(V / V_{\mathrm{o}}\right)$, where $V_{\mathrm{o}}=0.50 \mathrm{~V}$ (Fig. 3B). Apparently, a tunneling barrier dominates the $I-V$ of the innermost shells, most likely because these shells can only couple to the external electrodes through a barrier composed of many graphitic layers. For intermediate shells not in direct contact with the electrodes, the unusual shape of the measured $I-V$ curves can be understood qualitatively as the result of a depth-dependent barrier in series with a nanotube's intrinsic, longitudinal $I-V$ characteristic. This series barrier explains the gradual increase of the bias required to reach current saturation seen in Fig. 3. Furthermore, the transition from linear to nonlinear $I-V$ curves observed here and the abundance of similar nonlinear $I-V \mathrm{~s}$ reported previously suggests that transport experiments often do not directly contact the current-carrying carbon shell of a MWNT but rather contact partial or incomplete shells sometimes observed by transmission electron microscopy (13).

Our measurements confirm the varying nature of MWNT shells, quantitatively address the electronic barrier between these shells, and attempt to isolate the contribution of a single shell to the overall conductance. Until now, theory and experiment have been

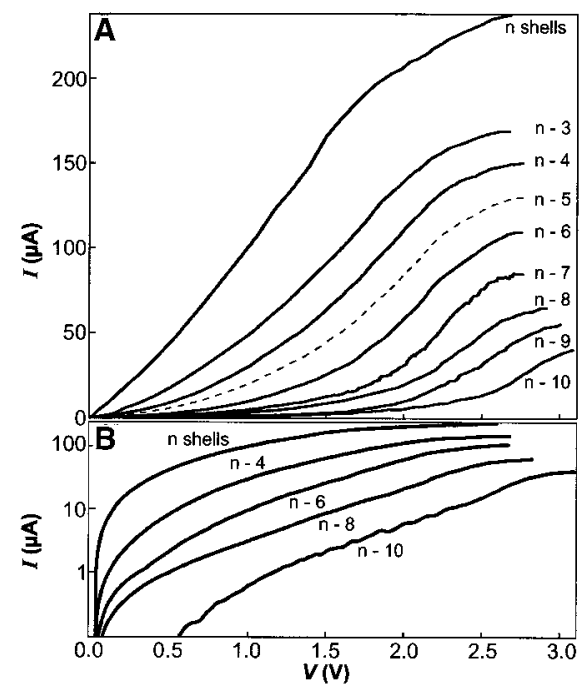

Fig. 3. (A) By recharacterizing the MWNT after the loss of individual shells, the contribution of each shell to the $I-V$ can be determined. On the basis of the uniform spacing of the sequence of $I-V$ curves, it appears that each shell saturates at the same current and that all of the shells contribute to conduction at moderate and high bias. The dashed line marks the estimated position of an $I-V$ curve that was not acquired. (B) A semilog plot of selected $I-V s$ indicates a tendency toward exponential $I-V s$ for the innermost shells due to the effective barrier between them and the external electrodes. Similar barriers likely play a role in all shells except the outermost one and help explain the nonlinear I-Vs reported for some MWNTs but not others. 
divided over these issues. MWNTs are too complex to create a realistic theoretical model; conversely, previous experiments had been unable to directly probe the inner carbon shells. The powerful technique of controlled breakdown introduced here has the potential to provide insight into the transport properties of these complex conductors.

Similar measurements have been performed on ropes of SWNTs. A SWNT rope differs from a MWNT in many consequential ways. First, each SWNT in a small rope can connect independently to the external electrodes. Thus, ropes, much more than MWNTs, may be modeled as independent, parallel conductors with total conductance $G\left(V_{\mathrm{g}}\right)=G_{\mathrm{m}}+G_{\mathrm{s}}\left(V_{\mathrm{g}}\right)$, where $G_{\mathrm{m}}$ is the contribution of the $\mathrm{m}$ nanotubes and $G_{\mathrm{s}}$ is the gate-dependent conductance of the s nanotubes (20). Second, multiple SWNTs within a rope are in contact with air, a potentially oxidizing environment, so many carbon shells can fail at once, rather than the uniform, shellby-shell failure observed in MWNTs. And lastly, the SWNTs within a small rope do not electrostatically shield each other as effectively as the concentric shells of a MWNT. As a result, the gate electrode is quite effective at depleting carriers from the s-SWNTs. In this state, current-induced oxidation can be directed solely at the m-SWNTs within the rope.

In Fig. 4, A and B depict $G\left(V_{\mathrm{g}}\right)$ for two small SWNT ropes before and after controlled breakdown. The unperturbed samples had a conductance that could be partially modulated by the gate electrode, much like the case for MWNTs. With $V_{\mathrm{g}}$ held at $+10 \mathrm{~V}$ to deplete the predominantly p-type s-SWNTs, a stress of several volts was applied to the rope. As the m-SWNTs in the rope were destroyed, $G_{\mathrm{m}}$ decreased to zero. In contrast, the modulating component $G_{\mathrm{s}}\left(V_{\mathrm{g}}\right)$ did not change. The constancy of $G_{\mathrm{s}}\left(V_{\mathrm{g}}\right)$ indicates that the depleted s-SWNTs were very effectively protected from damage. Further-

more, it suggests little electronic interaction between the different types of SWNTs. Measurements of the temperature dependence of $G_{\mathrm{s}}\left(V_{\mathrm{g}}\right)$ with and without metallic neighbors could address this issue of interactions and determine at what energy range, if any, such interactions become important (18).

To see how far this technique may be extended, we measured very thick ropes containing hundreds of SWNTs. Even these samples could be converted effectively into FETs (Fig. 4C). However, in this case the gradual decrease of $G_{\mathrm{m}}$ halted before it reached zero, presumably because m-SWNTs at the core of the rope were encased by s-SWNTs. The ultimate destruction of these weakly coupled m-SWNTs required higher voltages and a sacrifice of some of the surrounding s-SWNTs. As a result, a rope with many s-SWNTs and a large, initial modulation of $G_{\mathrm{s}}>10 \mu \mathrm{S}$ may only result in a FET with $G_{\mathrm{s}} \sim 1 \mu \mathrm{S}$.

The controlled breakdown technique is extremely valuable not only for the study of MWNTs and SWNT interactions but also for the fabrication of nanotube-based electronic devices. Until now, SWNT FETs have been fabricated individually. Typically, SWNTs in solution are spread on top of prefabricated electrode arrays. A very low surface coverage ensures that one SWNT at most connects a source and drain electrode. At this density, the great majority of circuits remain disconnected, but some incorporate an m-SWNT and others have an s-SWNT.

Although this technique has proven valuable for the initial characterization of SWNT properties, practical applications will surely require the reliable generation of many devices in parallel. Achieving densely packed FETs, for example, requires purely s-SWNTs at a high enough density to guarantee interconnections at all of the desired positions (whether grown in situ by chemical vapor deposition techniques or grown ex situ and deposited). Such high surface

Fig. 4. (A and B) Stressing a mixture of $\mathrm{s}$ - and m-SWNTs while simultaneously gating the bundle to deplete the semiconductors of carriers resulted in the selective breakdown of the $\mathrm{m}$ SWNTs. The $G\left(V_{g}\right)$ curve rigidly shifted downward as the $\mathrm{m}$-SWNTs were destroyed. The remaining current modulation is wholly due to the remaining sSWNTs. (C) In very thick ropes, some s-SWNTs must also be sacrificed to remove the innermost m-SWNTs. By combining this technique with standard lithography, arrays of three-terminal, nanotube-based FETs were created (D and E) out of disordered bundles containing both $\mathrm{m}$ - and s-SWNTs. Although these bun-
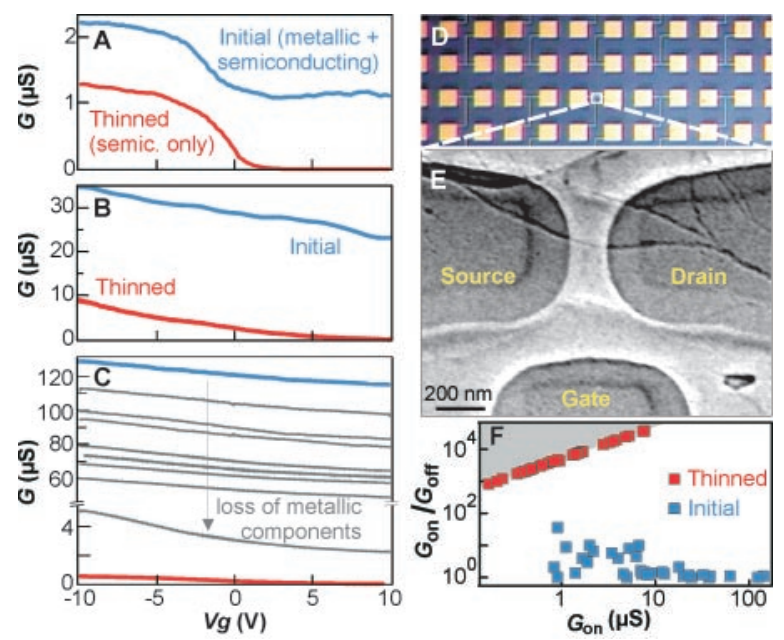

dles initially show little or no switching because of their metallic constituents, final devices with good FET characteristics were reliably achieved (F). densities favor multiple SWNTs and SWNT bundles that, because of the variability of SWNT properties, are dominated by m-tubes and are useless as s-channels. At present, no method exists for the synthesis of purely sSWNTs or for the separation of s-SWNTs from SWNT mixtures.

To demonstrate the potential of the controlled breakdown technique to solve these issues, we fabricated small arrays of independently addressable SWNT FETs. Using standard lithography, an array of electrodes (source, drain, and gate) was defined on top of a dilute film of SWNT ropes (Fig. 4, D and E). The SWNT density was adjusted to ensure that at least one rope shorted every set of electrodes while minimizing the unwanted connections between devices. Ropes between source and drain electrodes were converted into FETs by selective breakdown, and stray connections were removed entirely by complete breakdown.

Our preliminary results indicate that the generation of SWNT FETs can be achieved with greater than $90 \%$ certainty from this disordered starting material (21). Figure 4F summarizes results for 32 devices incorporating one or more SWNT ropes. Before modification, the rope conductances varied widely due to the distribution of rope sizes as well as contact effects, and very few devices could be substantially depleted by the gate. Upon breaking the m-SWNTs, the conductance of each rope decreased but the remaining channel was solely semiconducting and could be fully depleted. The resulting devices have reasonable FET characteristics with switching ratios limited primarily by contact resistances, a difficulty to be addressed separately. We anticipate that the multiple, small SWNT bundles, which can be produced by chemical vapor growth, may alleviate the difficulties encountered with large bundles and result in FETs having excellent conductivities and switching ratios.

Although this study focused on the particular system of carbon nanotubes, the same principles may apply broadly to various molecular electronics systems. We have shown

Table 1. Comparison of relative band gaps from experiments (Fig. 2B) with calculations based on the expected diameter dependence. The only parameters are the initial diameter of the tube and the $0.34 \mathrm{~nm}$ spacing between adjacent shells. Calc., calculated; Meas., measured.

\begin{tabular}{lccc}
\hline & \multirow{2}{l}{$\begin{array}{c}\text { Diameter } \\
\text { Shell }\end{array}$} & \multicolumn{2}{c}{ Relative band gap (eV) } \\
\cline { 3 - 4 } & & Calc. & Meas. \\
\hline $\mathrm{n}$ & 9.5 & & \\
$\sim$ & $\sim$ & & \\
$\mathrm{n}-10$ & 2.7 & 0.24 & 0.22 \\
$\mathrm{n}-11$ & 2.0 & 0.33 & \\
$\mathrm{n}-12$ & 1.3 & 0.49 & 0.48 \\
$\mathrm{n}-13$ & 0.7 & 1.00 & 1.00 \\
\hline
\end{tabular}


that arrays of molecular devices can be produced by design with the use of external electrical means and without a need for actual control at the nanometer scale. Discretionary modification allowed the definition of useful electronic elements from a random mixture. Though we have applied this solution to solve the problem of variation inherent to carbon nanotubes, one could imagine generating similar results using mixtures of carefully chosen molecules. In that more general sense, the possibilities for active circuitry have yet to be explored.

References and Notes

1. H. Dai, E. W. Wong, C. M. Lieber, Science 272, 523 (1996).

2. J. E. Fischer et al., Phys. Rev. B 55, R4921 (1997).

3. S. J. Tans, A. R. M. Verschueren, C. Dekker, Nature 393, 49 (1998)

4. R. Martel, T. Schmidt, H. R. Shea, T. Hertel, Ph. Avouris, Appl. Phys. Lett. 73, 2447 (1998).

5. L. Chico, V. H. Crespi, L. X. Benedict, S. G. Louie, M. L. Cohen, Phys. Rev. Lett. 76, 971 (1996).

6. R. Saito, G. Dresselhaus, M. S. Dresselhaus, Phys. Rev. B 53, 2044 (1996)

7. MWNTs for this study were provided by W. deHeer and were grown by standard arc techniques. SWNTs were provided by R. Smalley and were grown by metal-catalyzed laser ablation. Both types of nanotubes were dispersed in dichloroethane and purified solely by centrifugation.

8. P. G. Collins, M. Arnold, M. Hersam, R. Martel, Ph. Avouris, Phys. Rev. Lett. 86, 3128 (2001).

9. R. Saito, G. Dresselhaus, M. S. Dresselhaus, J. Appl. Phys. 73, 494 (1993).

10. A. Bachtold, et al., Nature 397, 673 (1999).

11. S. Frank, P. Poncharal, Z. L. Wang, W. A. de Heer, Science 280, 1744 (1998).

12. C. Schöenenberger, A. Bachtold, C. Strunk, J.-P. Salvetat, L. Forro, Appl. Phys. A 69, 283 (1999).

13. S. lijima, Nature 354, 56 (1991).

14. C.-H. Kiang, M. Endo, P. M. Ajayan, G. Dresselhaus, M. S. Dresselhaus, Phys. Rev. Lett. 81, 1869 (1998).

15. The use of high-voltage pulses to break shells leads to some rearrangement of trapped charges on the underlying $\mathrm{SiO}_{2}$ substrate. To simplify the comparison among shells, we centered each curve in Fig. $2 \mathrm{~B}$ near $V_{\mathrm{g}}=0$.

16. J. W. Mintmire, B. I. Dunlap, C. T. White, Phys. Rev. Lett. 68, 631 (1992).

17. R. Saito, M. Fujita, G. Dresselhaus, M. S. Dresselhaus, Phys. Rev. B 46, 1804 (1992).

18. High-bias $I-V$ curves must be acquired in high vacuum to suppress destructive oxidation. Between each curve, the MWNT was exposed to air in order to remove single carbon shells in a controlled manner. Four- and two-probe measurements are periodically compared to monitor the contact resistance $R_{\mathrm{c}}$ to each nanotube. The data shown here are for samples exhibiting constant $R_{\mathrm{c}}$ of a few kilohms throughout the series of measurements. Samples with high $R_{\mathrm{c}}$ tend to fail at the contact, as opposed to the shellby-shell mechanism described here.

19. Z. Yao, C. L. Kane, C. Dekker, Phys. Rev. Lett. 84, 2941 (2000).

20. For example, see H. Stahl, J. Appenzeller, R. Martel, Ph. Avouris, B. Lengeler, Phys. Rev. Lett. 85, 5186 (2000).

21. The remaining $10 \%$ form open circuits before we can achieve complete switching. Imaging these samples typically reveals large contaminant particles or multirope crossings, which likely result in weak, dissipative links.

22. We thank R. Martel for valuable discussions, K. J. Chan for assistance with lithography, and B. Ek for expert technical assistance. M.A. acknowledges the support of an IBM summer internship.

4 January 2001; accepted 5 March 2001

\title{
Selective Bond Dissociation and Rearrangement with Optimally Tailored, Strong-Field Laser Pulses
}

\author{
Robert J. Levis, ${ }^{1 *}$ Getahun M. Menkir, ${ }^{1}$ Herschel Rabitz ${ }^{2}$
}

\begin{abstract}
We used strong-field laser pulses that were tailored with closed-loop optimal control to govern specified chemical dissociation and reactivity channels in a series of organic molecules. Selective cleavage and rearrangement of chemical bonds having dissociation energies up to approximately 100 kilocalories per mole (about 4 electron volts) are reported for polyatomic molecules, including $\left(\mathrm{CH}_{3}\right)_{2} \mathrm{CO}$ (acetone), $\mathrm{CH}_{3} \mathrm{COCF}_{3}$ (trifluoroacetone), and $\mathrm{C}_{6} \mathrm{H}_{5} \mathrm{COCH}_{3}$ (acetophenone). Control over the formation of $\mathrm{CH}_{3} \mathrm{CO}$ from $\left(\mathrm{CH}_{3}\right)_{2} \mathrm{CO}, \mathrm{CF}_{3}\left(\right.$ or $\left.\mathrm{CH}_{3}\right)$ from $\mathrm{CH}_{3} \mathrm{COCF}_{3}$, and $\mathrm{C}_{6} \mathrm{H}_{5} \mathrm{CH}_{3}$ (toluene) from $\mathrm{C}_{6} \mathrm{H}_{5} \mathrm{COCH}_{3}$ was observed with high selectivity. Strong-field control appears to have generic applicability for manipulating molecular reactivity because the tailored intense laser fields (about $10^{13}$ watts per square centimeter) can dynamically Stark shift many excited states into resonance, and consequently, the method is not confined by resonant spectral restrictions found in the perturbative (weak-field) regime.
\end{abstract}

The control of organic chemistry with optical fields has been a long-sought-after goal. This objective goes beyond traditional photochemistry, where variation in the product distribution is obtained by tuning a monochromatic radiation source to excited states of a precursor molecule. Here, we focus on whether a suitably tailored laser pulse can guide a distribution of products to a specific channel, characterized by dissociation or rearrangement, as illustrated in Fig. 1. Successful control in this context implies cooperative interaction between a (perhaps complex) timedependent electric field of the laser pulse and the precursor molecule's dynamics to alter the product distribution in a desirable and variable manner. The objective is to maximize the yield of a specified product by means of a suitable closed-loop laboratory learning procedure that determines the optimal laser pulse. The capability of controlling reactivity, especially of organic species, by this means could have implications in diverse industrial and biological or medicinal settings. For example, microelectronic lithography, the fabrication of gene chips, and photodynamic therapy could all benefit from highly controllable photochemistry.

Since the introduction of the concept of teaching lasers to control photodynamics with closed-loop methods (1), the process has been used for optimization of laser-induced fluorescence (2), stimulated Raman emission from molecules (3), high harmonic generation (4), ultrafast semiconductor switching

${ }^{1}$ Department of Chemistry, Wayne State University, Detroit, MI 48202, USA. 'Department of Chemistry, Princeton University, Princeton, NJ 08540, USA.

*To whom correspondence should be addressed.
(5), and management of pulse propagation through nonlinear media (6). The first implementation of this scheme for dissociating bonds was demonstrated in groundbreaking work on organometallic systems (7) and has since been demonstrated in the cleavage of weakly bound cluster species (8). The control of organic reactivity, where bond strengths exceed $\sim 50 \mathrm{kcal} / \mathrm{mol}$, remains an open and important area of optical control research.

We demonstrate a potentially universal, ultrafast laser excitation procedure to achieve control over molecular reactivity. This procedure is based on closed-loop learning of optimally shaped, strong-field, near-infrared pulses (centered around $800 \mathrm{~nm}$ ) guided just by the desired product. A strong-field laser pulse $\left(\sim 10^{13} \mathrm{~W} \mathrm{~cm}^{-2}\right)$ implies that the associated electric field magnitude is on the order of the valence electron's binding energy in the molecule. This magnitude is a critical enabling feature for broad-scale application upon consideration of the bandwidth required for the effective control of chemical reactions. Weak-field, continuous wave pulses must be precisely tuned to a molecular resonance for coupling; Fig. 2A represents such an excitation scheme for most conventional photochemical reactions. Ultrafast laser systems allow $\sim 0.1 \mathrm{eV}$ of bandwidth for a 25 -fs pulse (for example, 775 to $825 \mathrm{~nm}$ for a near-infrared pulse). The relatively small bandwidth around the laser carrier frequency limits control in the weak-field case (Fig. 2B). However, with strong-field pulses, the nominal eigenstates of the molecule can be placed into resonance through a combination of Stark shifting (up to $\sim 10 \mathrm{eV}$ in our experiment) (9) and multiphoton excitation (10 to 50 photons using the Ti:sapphire laser cen- 